\title{
Reconstruction of few-fs XUV pulses with a perturbative approach
}

\author{
Bruno Moio ${ }^{1,2, *}$,Fabio Medeghini 1,2, Gian Luca Dolso ${ }^{1,}$, Giacomo Inzani ${ }^{1,}$, Nicola Di Palo ${ }^{1,2,}$, Rocío Borrego-Varillas², \\ Mauro Nisoli ${ }^{1,2}$, and Matteo Lucchini ${ }^{1,2}$, \\ ${ }^{1}$ Department of Physics, Politecnico di Milano, 20133 Milano, Italy \\ ${ }^{2}$ Institute for Photonics and Nanotechnologies, IFN-CNR, 20133 Milano, Italy
}

\begin{abstract}
A precise temporal characterization of the pulses involved in pump-probe experiments is crucial for a proper investigation of the ultrafast dynamics in several physical systems. Indeed, it is required for the assessment of the dynamical properties under examination with sufficient temporal resolution. In the fewfs/attosecond domain, typical reconstruction procedures require time-consuming interative methods, which are also sensitive to the experimental noise and to the distortion of the measurement. We developed an approach, called Simplified Trace Reconstruction In the Perturbative regimE (STRIPE), which allows us for a precise characterization of the infrared (IR) and extreme ultraviolet (XUV) pulses, used in a pump-probe experiment. Our method is not based on a phase retrival algorithm, and for this it is typically much faster than the other ones currently known. Moreover, it allows for easily including in the reconstruction the experimental non-idealities that may affect the measurement, like possible distortion due to the measurement procedure itself.
\end{abstract}

\section{Introduction}

Recently, ultrashort extreme-ultraviolet (XUV) pulses, combined with few-cycle infrared (IR) radiation in a pump-probe scheme, open the possibility of studying dynamics in a wide range of systems, from molecules to solid stated materials $[1,2]$. In addition, the possibility to select single harmonics from a much wider XUV spectrum [3] allows to selectively access single transitions, but maintaining at the sane time the possibility to tune the spectral range to investigate.

The use of single-harmonic extreme-ultraviolet radiation has been widely employed for the investigation of electron dynamics in molecules [4] with excellent simultaneous temporal and spectral resolution. The availability of few-fs single harmonic XUV pulses allowed to study the ultrafast conical-intersection dynamics in $\mathrm{NO}_{2}$ [5] and the temporal evolution of ethylene cation with state-to-state resolution [6]. A proper analysis of such dynamics calls for an accurate characterization of the pulses. A commonly used method consists in measuring several photoelectron spectra, as produced by the XUV radiation in the presence of a delayed infrared pulse, and employing a phase retrival algorithm, based on iterative procedures, for charachterizing the pulses involved [7,8]. These phase retrival algorithm are typically computationally expensive and require a very clean experimental measurement, since are sensitive the residual noise of the trace.

In this work, we propose a novel approach for the pulse temporal characterization, based on a simplified method called Simplified Trace Reconstruction In the Perturbative regimE (STRIPE). Our method is much faster than the re-

*e-mail: bruno.moio@polimi.it trieval algorithms currently used and can be applied directly to differential phototelectron traces, to remove the distorsions that may arise from background signals. Moreover, our method allows for a proper pulse characterization even if the signal to noise ratio is extremely poor. This makes the measurement and the reconstruction inherently robust with respect to noise. Finally, being more versatile than a standard iterative algorithm, STRIPE can be easily modified to explicitly include the system non-idealities due to the meaurement procedure itself, like electronic peak broadening.

\section{The STRIPE model}

In the strong field approximation (SFA) [9], the photoionazion process of a rare gas, in the presence of XUV and IR pulses can be described by the following formula (atomic units are used hereafter):

$$
S(\mathbf{p}, \tau)=\left|\int_{-\infty}^{+\infty} d t E_{X U V}(t+\tau) e^{i \phi(\mathbf{p}, t)} e^{i\left(\frac{p^{2}}{2}+I_{p}\right) t}\right|^{2}
$$

where $E_{X U V}(t)$ is the XUV pulse, $\phi(\mathbf{p}, t)$ is a phase term which accounts for the effect of the IR field on the photoelectron wavepacket of momentum $\mathbf{p}, I_{p}$ is the atomic ionization potential and $\tau$ is the relative delay between the XUV and IR. We can now develop the simplified model by applying a series of approximations to the Eq. (1). In particular, under the central momentum approximation (CMA), the perturbative approximation and the slowlyvarying envelope approximation (SVEA), we can reformulate the previous equation into the following expression 
[10]:

$$
S(\omega, \tau) \simeq\left|\int_{-\infty}^{+\infty} d t E_{X U V}(t+\tau) e^{i \frac{p_{c}}{\omega_{0}^{2}} E_{I R}(t)} e^{i \omega t}\right|^{2}
$$

where we set $\omega=p^{2} / 2+I_{p}$. Equation (2) represents the model upon which STRIPE is based. Figure 1 shows

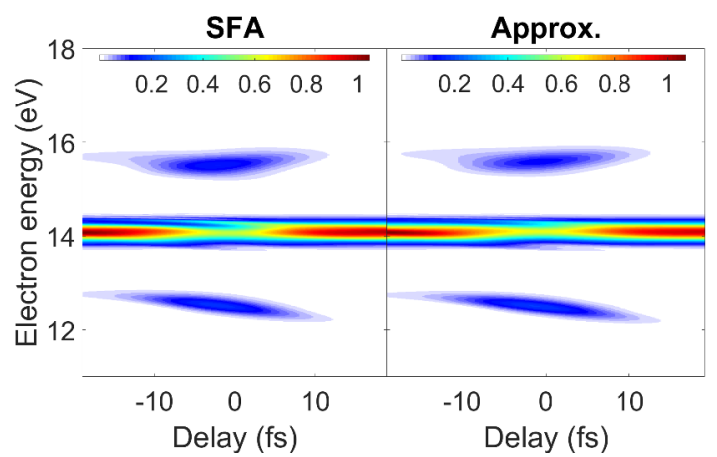

Figure 1. Comparison of a single-harmonic phototelectron trace, computed with the SFA formula (left) and the STRIPE model (right). The agreement is very good as the STRIPE model can describe all the features of the main band and the sidebands. However, the computational effort for computing the trace with the simplified model is significantly smaller than the one needed for the SFA calculations.

a comparison between a single-harmonic phototelectron trace, computed with the full SFA model (left panel) and one computed with the STRIPE formula (right panel). We considered an XUV and IR pulses, with durations of about 10 and $9 \mathrm{fs}$, respectively, a second order dispersion of 10 and $20 \mathrm{fs}^{2}$, a third order of 300 and $30 \mathrm{fs}^{3}$ and a fourth order of 2500 and $-150 \mathrm{fs}^{4}$. The IR intensity is $5 \cdot 10^{11}$ $\mathrm{W} / \mathrm{cm}^{2}$.The parameters are deliberately set at the limits of validity of the approximated model, in order to test the procedure in the worst-case scenario.

The agreement between the two simulations is very good, as the STRIPE model can reproduce all the main features observed in the SFA trace. Its worth mentioning that the computational effort to compute the STRIPE trace is much smaller than the one needed for the SFA calculations. Indeed, the time to compute a single typical SFA trace amounts to about 30 seconds, against the 40 milliseconds needed for the computaton with the STRIPE model.

\section{Trace reconstruction}

If, on one side, it is clear that the approximated model produces traces that nicely compare to the SFA calculations, on the other, to prove the possibility to use the STRIPE model for the characterization of XUV and IR pulses in photoelectron experiments, we must apply it to some simulated traces, and see if it can recover the fields involved. We start by using STRIPE in a reconstruction of a clean trace, with noise, to first test the method. Then, we repeated the reconstruction also including noise, to prove the real applicability of the algorithm. Eventually, we tested the case of a real experimental trace.

\subsection{Reconstruction with no noise}

As a first attempt, we applied STRIPE for the reconstruction of pulses out of a simulated trace computed with SFA calculations, where no approximations are considered, and with no additional noise introduced. The reconstruction is done by a non-linear fitting procedure, employing the simplified model in Eq. (2). The trace on which we applied our algorithm is the one in the left panel of Fig. 1 , whereas the pulses retrieved by STRIPE are shown in Fig. 2 (red dashed lines), together with the ones used in the SFA simulations (black lines). Both the XUV and IR
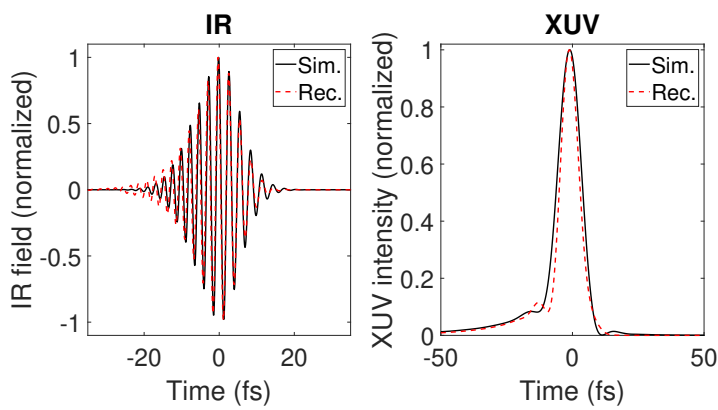

Figure 2. Result of the STRIPE reconstruction, applied to the trace simulated with SFA in Figure 1. The black solid lines show the simulated IR and XUV pulses, whereas the dashed red lines show the reconstructed ones.

pulses are correctly retrieved, with small deviations from the ones used in the simulation, despite the parameters are chosen to stretch as much as possible the approximations of the method. We must mention that an SHS experiment is not sensitive to the phase of the IR field. In this case, we chose the IR phase to match the one of the simulation, just to highlight the agreement between the simulated and retrieved pulse energy dispersion. In any case, this result proves that, despite the fact that the model upon which it is based is heavily approximated, STRIPE can serve as a useful and efficient tool for pulse characterization.

\subsection{Reconstruction with white noise}

Another important feature of our reconstruction method is a great robustness against the experimental noise. To prove this claim, we applied STRIPE to a trace, where an additional very large white noise is added. We choose an extreme noise level, much larger than in any reasonable experiment, to prove that our method is basically insensitive to any noise intensity. The noisy trace is shown in the top panel of Fig. 3, whereas the comparison between the simulated and reconstructed fields is displayed in the bottom left and right panels of the same figure. Also here we set the IR phase to match the simulated value, for a clearer visualization. The comparison shows that, despite the trace being completely distorted by the noise, with a signal-to-noise level ratio is unrealistically low, the information is not actually lost and STRIPE can access it, with little to no additional uncertainty, as one can see by comparing the fields if Figs. 2 and 3. Indeed, the precision 

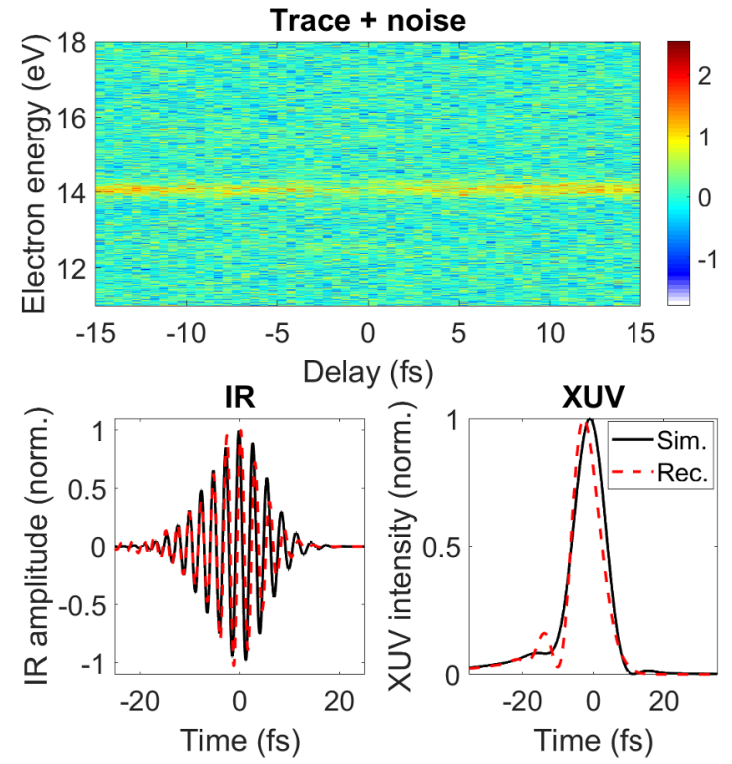

Figure 3. Results of the STRIPE reconstruction, with a large white noise applied to the SFA trace. The top panel shows the noisy simulated trace, whereas the bottom panels show a comparison of the simulated and reconstructed pulse. Despite the signal-to-noise ratio is very low, STRIPE can recover the pulses with almost the same accuracy.

of the field reconstruction is comparable to the case with no noise, showing that the trace distortion is not basically affecting the robustness of the algorithm.

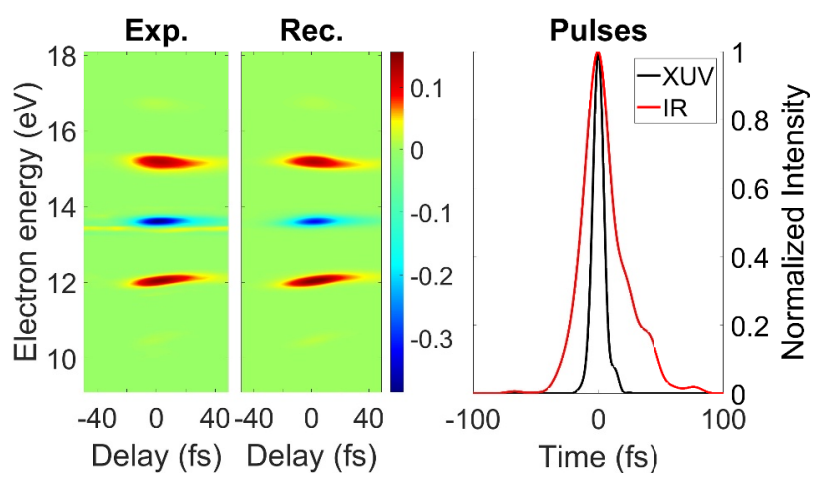

Figure 4. Experimental and reconstruced single-harmonic differential traces. Despite the simplified model, STRIPE can catch all the features of the experimetnal trace. On the right, we show the reconstructed XUV (black) and IR (red) pulses.

\subsection{Reconstruction with experimental trace}

To undoubtedly prove that our method can be applied to real-case measurements, we performed the reconstruction on an experimental trace. We carried out the retrieval on the differential trace, obtained by subtracting the XUVonly spectrum, to remove the effect of a background signal in the experiments, and to include in the fitting the artificial spectral broadening due to the electronic response of the acquisition system. Figure 4 shows a comparison between the experimental differential trace and the reconstruction one, and the reconstructed XUV (black line) and IR (red line) pulses. The reconstruction is very accurate, and all the features of the trace are correctly recovered. Moreover, the retrieval is not affected by the experimental noise. The extracted IR field duration of 26.9 fs compares very well to the one obtained with an independent FROG measurement, while for the XUV STRIPE gives a duration of $10.2 \mathrm{fs}$, in line with what expected from ray-tracing calculations.

\section{Conclusions}

We presented STRIPE, a novel method for the characterization of XUV and IR femtosecond pulses in a photoelectron experiment. Our method allows to reconstruct the pulses in a much faster way and with high degree of accuracy, despite all the approximation introduced. The reconstruction performed on a very noisy trace shows that STRIPE is intrinsically robust against any level of noise, and by applying it to an experimental trace affected by large non idealities, we proved this method to be capable of precise retrieval also in real, extreme cases. Indeed, the reconstructed pulses agree with what obtained with independent measurements. So far we tested our method only with single-harmonic spectrograms, but we already observed that the model in Eq. (2) can also be used to properly simulated photoelectron traces in a variety of settings (streaking, RABBITT, etc.), making our technique a valuable tool for a fast characterization of the pulses in a wide range of experiments.

\section{References}

[1] Krausz, F., and Misha I., Rev. Mod. Phys. 81, 163 (2009)

[2] Calegari, F., et al., Science 346 336-339 (2014)

[3] Poletto, L., et al., Opt. Lett. 32 2897-2899 (2007)

[4] Eckstein, M., et al., J. Phys. Chem. Lett. 6, 419-425 (2015)

[5] von Conta, A., et al., Nat. Commun. 9, 1-10 (2018)

[6] Lucchini, M., et al. CLEO/Europe-EQEC, (2019)

[7] Lucchini, M., et al., Opt. Expr. 26, 6771-6784 (2018)

[8] Murari, M., et al., Opt. Expr. 28, 10210-10224 (2020)

[9] Kitzler, M. et al., Phys. Rev. Lett. 88, 173904 (2002)

[10] Moio, B. et al., J. Phys. B: At. Mol. Opt. Phys. 54, 154003 (2021) 